\title{
Efimov Physics and the Three-Body Parameter within a Two-Channel Framework
}

\author{
P. K. Sørensen, D. V. Fedorov, A. S. Jensen, and N. T. Zinner \\ Department of Physics and Astronomy, Aarhus University, DK-8000 Aarhus C, Denmark
}

(Dated: July 2, 2018)

\begin{abstract}
We calculate shallow three-body bound states in the universal regime, defined by Efimov, with inclusion of both scattering length and effective range parameters. We find corrections to the universal scaling laws for large binding energies. For narrow resonances we find a distinct nonmonotonic behavior of the threshold at which the lowest Efimov trimer merges with the threebody continuum. The origin of the three-body parameter is related to the two-body atom-atom interactions in a physically clear model. Our results demonstrate that experimental information from narrow Feshbach resonances and/or mixed systems are of vital importance to pin down the relation of two- and three-body physics in atomic systems.
\end{abstract}

PACS numbers: 03.65.Ge,31.15.ac, 21.45.-v, 67.85.-d

Introduction. The counterintuitive behavior of threebody systems at the threshold for two-body binding is highlighted by the Efimov effect where an infinitude of geometrically scaling states appears [1]. While unsuccesfully sought for in nuclear physics [2], the effect has been confirmed and explored in ultracold atomic gases [3]. From the theoretical point of view these systems have been described accurately by universal theories that only take the lowest order scattering dynamics into account through the two-body scattering length, $a[4,[5]$. However, the overall scale of the spectrum cannot be obtained in the universal theory and the so-called three-body parameter, $\Lambda$, is needed to complete the formalism.

In ultracold atomic gases, trimer physics can be studied using inter-atomic Feshbach resonances [6] that provide tunability of $a$ over many orders of magnitude. The parameters of these resonances will in general depend on the microscopic details of a particular atomic system. Still, in a surprising development, the Grimm group have reported strong indications that the three-body parameter is determined by the van der Waals length, $r_{\mathrm{vdW}}$ [7]. This implies that atomic trimer physics in the weakly bound limit only depend on two-body parameters. The experimental findings have generated a flurry of recent theoretical interest [8 11]. It has been suggested that the presence of many deep bound states in the two-body potential typical of alkali atom systems will suppress the dependence on short-range physics due to a large inner repulsive barrier [9, 10].

The most natural way to eliminate any need for a three-body parameter is to use a finite-range two-body potential that exhibits the known features of the interatomic interaction. Here we pursue this natural and straightforward approach to the issue of the origin of $\Lambda$ in three-body physics. As three-body calculations with finite-range potentials are typically very cumbersome, it is highly desirable to have simple models that mimic the finite-range physics. Therefore we consider here a model with zero-range interactions that includes finite-range correction effects. Consequently, we solve the threebody Schrödinger equation using one- and two-channel models for the description of the Feshbach resonances

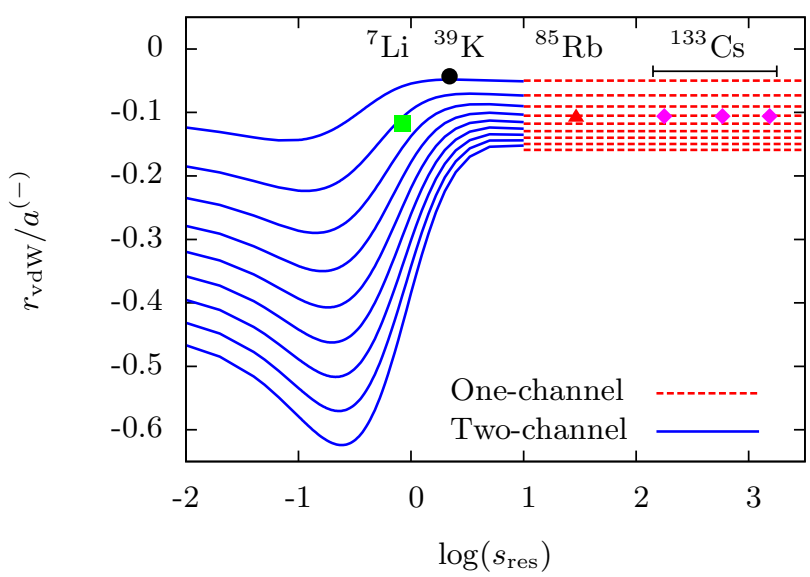

FIG. 1: (Color online) The threshold scattering length $a^{(-)}$ at which the lowest universal Efimov trimer merges with the three-atom continuum plotted against the strength, $s_{\text {res }}$, of the Feshbach resonance. The right-hand side corresponds to broad resonances. The different curves of like linetype show result with different three-body parameters, $\rho_{c}$, in units of the atomic van der Waals length, $r_{\mathrm{vdW}}$. The values of $\rho_{c}$ decrease from top to bottom (precise values are given in the text). Experimental values are from ${ }^{133} \mathrm{Cs}$ [7, 12], ${ }^{7} \mathrm{Li}$ [13], ${ }^{39} \mathrm{~K}[14]$, and ${ }^{85} \mathrm{Rb}[15]$.

that are used to control interactions in experiments [ 6 ]. The three-body parameter is considered parametrically in both models, and this allows us to study the threshold behavior of Efimov trimers as function of $\Lambda$ and the width of the Fesbhach resonance which is related to the effective range, $r_{e}\left[\underline{6}\right.$ ]. Here $r_{\mathrm{vdW}}$ and $r_{e}$ are intimately connected through semiclassical calculations [16 18]. It is conceivable that this explains the observed values of $\Lambda$ as $r_{e}$ or $r_{\mathrm{vdW}}$ provides a background scale that determines the overall scale. However, given the delicate non-classical nature of the universal trimer states it is far from obvious if and how this can work out.

The experimental findings indicate that for several different atomic species the ratio of threshold scattering 
length $a^{(-)}$for creation of Efimov trimers out of the three-body continuum is of order $a^{(-)} / r_{\mathrm{vdW}}=8.5 \sim 9.5$ [9]. Here we show that this result can be obtained in a single-channel zero-range model for a specific choice of $\Lambda$, which can be related to the underlying two-body atomatom physics in a natural way. Within a two-channel model we find an intriguing non-trivial behavior of $a^{(-)}$ for narrow resonances, irrespective of the three-body parameter, see Fig. 1. We also study the behavior of $a^{(-)}$ as a function of the number of bound states allowed by the two-body atomic potential for both one- and twochannel models. Generally, we find that the inclusion of effective range decreases $\left|a^{(-)}\right|$. Our results predict that narrow resonance systems are important for obtaining a full picture of the relation between two- and three-body parameters for universal bound state physics.

Method. We consider a system of three identical bosonic particles using hyperspherical coordinates [19] defined from the Cartesian coordinates $\boldsymbol{r}_{i}, \boldsymbol{r}_{j}, \boldsymbol{r}_{k}$ through $\boldsymbol{x}_{i}=\left(\boldsymbol{r}_{j}-\boldsymbol{r}_{k}\right) / \sqrt{2}$ and $\boldsymbol{y}_{i}=\frac{2}{3}\left(\boldsymbol{r}_{i}-\left(\boldsymbol{r}_{j}+\boldsymbol{r}_{k}\right) / 2\right)$ as hyperradius, $\rho=\sqrt{\boldsymbol{x}_{i}^{2}+\boldsymbol{y}_{i}^{2}}$, and hyperangle, $\alpha_{i}=\tan ^{-1} \frac{\left|\boldsymbol{x}_{i}\right|}{\left|\boldsymbol{y}_{i}\right|}$. $\{i, j, k\}$ are cyclic permutations of $\{1,2,3\}$ and $\rho$ is independent of this choice. We apply the hyperspherical adiabatic approach with wave function $\Psi(\rho, \Omega)=$ $\rho^{-5 / 2} \sum_{n} f_{n}(\rho) \Phi_{n}(\rho, \Omega)$, where $\Omega=\left\{\alpha_{i}, \boldsymbol{x}_{i} /\left|\boldsymbol{x}_{i}\right|, \boldsymbol{y}_{i} /\left|\boldsymbol{y}_{i}\right|\right\}$ is a set of angular coordinates. We keep only the lowest adiabatic potential corresponding to $n=0$, and index $n$ is henceforth suppressed. For the description of Efimov trimer states, this approximation has proven extremely accurate [4]. The radial equation is

$$
\left(-\frac{d^{2}}{d \rho^{2}}+\frac{\lambda(\rho)+15 / 4}{\rho^{2}}-\frac{2 m E}{\hbar^{2}}\right) f(\rho)=0,
$$

where $\lambda(\rho)$ is the eigenvalue to the hyperangular equation

$$
\left(\tilde{\Lambda}^{2}+\frac{2 m \rho^{2}}{\hbar^{2}} V\right) \Phi(\rho, \Omega)=\lambda(\rho) \Phi(\rho, \Omega)
$$

in which $\tilde{\Lambda}^{2}$ is the generalized angular momentum operator, $V$ is the two-particle interaction potentials, and $m$ is the atomic mass. In Eq. (1), the non-adiabatic corrections are omitted as they are found to be negligible.

We use zero-range single-channel and a two-channel interaction models [20]. The former is accurate for broad Feshbach resonances (small $r_{e}$ ) and the latter for narrow resonances (large $r_{e}$ ). Both models contain the background scattering length in the open channel, $a_{\text {open }}$. Our model must predict three-body properties solely from two-body potentials, so we must insist that $a_{\text {open }}$ is a quantity most naturally associated to the two-body $r_{\mathrm{vdW}}$. In a semiclassical approach, the scattering length of such potentials is $\bar{a} \equiv 4 \pi(\Gamma[1 / 4])^{-2} r_{\mathrm{vdW}} \approx 0.956 r_{\mathrm{vdW}}[16]$. Then $a_{\text {open }}$ should be identified with $r_{\mathrm{vdW}}$ to within a few percent. The resonance strength is related to $r_{e}$ [20]. We characterize the strength by $s_{\text {res }}=r_{\mathrm{vdW}} /\left|R_{0}\right|$, where $R_{0}$ is the effective range when $|a|=\infty[6]$.

Eq. (1) is solved numerically for $f(\rho)$ with the condition $f\left(\rho_{\mathrm{L}}\right)=0$ for large $\rho_{\mathrm{L}}$. At short distance the

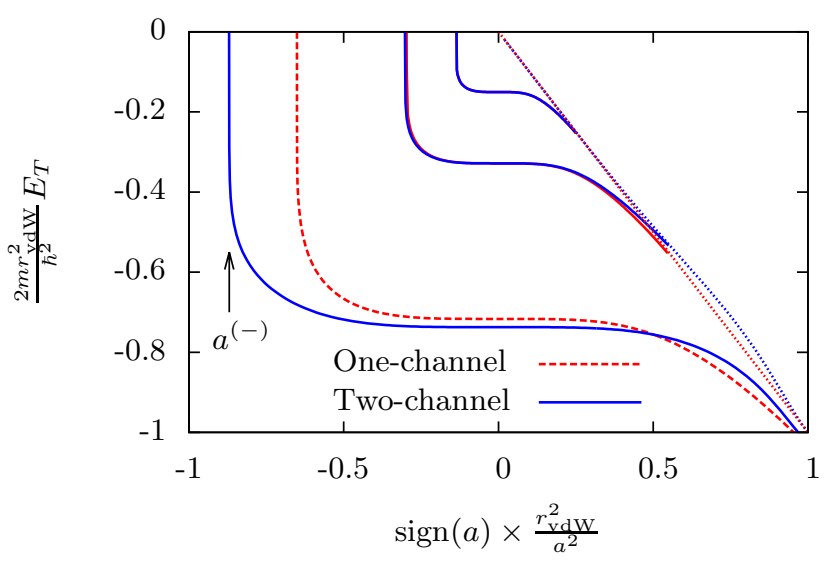

FIG. 2: (Color online) Trimer binding energy $E_{T}$ vs. scattering length $a$ for the one- and two-channel models with effective range $r_{e}=-5 r_{\mathrm{vdW}} \cdot a^{(-)}$is the threshold scattering length for existence of the lowest trimer. Finely dotted lines indicate atom-dimer threshold for positive $a$. Successive states have a scaling of 515 [1] and for visibility the axes are scaled by the power $1 / 8$.

zero-range models used here require a cut-off, $\rho_{c}$, with $f\left(\rho_{c}\right)=0$ [4]. Initially, we consider $\rho_{c}$ a parameter and study the three-body spectrum for different $\rho_{c}$ and different $s_{\text {res }} . \rho_{c}$ is the coordinate-space equivalent of $\Lambda$. Below we relate the $\rho_{c}$ to the two-body atomic potential.

Results. In Fig. 2 we show trimer energies, $E_{T}$, as function of $a$ for both one- and two-channel models. We find that the two-channel energies are generally lowest when $a<0$ and for $a>0$ the trimers cross before merging with the atom-dimer continuum. As indicated in Fig. 2, $a^{(-)}$is the threshold scattering length for appearance of the lowest Efimov trimer on the negative $a$ side. The two-channel model is here seen to move this threshold to the left, i.e. to smaller negative $a^{(-)}$. These thresholds and the energy for $|a|=\infty$ are connected by universal relations, where lower energy on resonance translates to smaller $\left|a^{(-)}\right|$at the threshold [1].

A systematic study of the influence of both $\rho_{c}$ and $r_{e}$ (or equivalently $s_{\text {res }}$ ) is shown in Fig. 1 which is one of our main results. The values of $\rho_{c} / r_{\mathrm{vdW}}$ from top to bottom in Fig. 1 are 1.20, 0.82, 0.66, 0.58, 0.51, 0.47, $0.42,0.40$, and 0.38 . They correspond to $n=0$ to 9 in Eq. (4) below. Both models agree for $s_{\text {res }} \gg 1$ and we plot the two-channel model results only in the region where it deviates. We find that to reproduce the experimental data for $s_{\text {res }} \gg 1$, a cut-off of $\rho_{c} / r_{\mathrm{vdW}}=0.58$ is required. However, for small $s_{\text {res }}$, the same cut-off does not reproduce the known data point coming from ${ }^{7} \mathrm{Li}$ (other measurements have smaller $a^{(-)}$[21, 22], which is closer to our predictions). While we do find an increase toward the ${ }^{39} \mathrm{~K}$ data point at small $s_{\text {res }}$, it cannot be accomodated for the same $\rho_{c}$.

In general, we find that, irrespective of $\rho_{c}$, the inclusion of effective range brings a non-monotonic behavior 
to $a^{(-)}$, and it tends to push the value of $a^{(-)}$down for small $s_{\text {res }}$ or large $\left|r_{e}\right|$. The reason can be seen in Fig. 6] where the adiabatic potentials for different effective ranges, $r_{e} / r_{\mathrm{vdw}}=0,-0.1,-1,-5$, are shown. At $r_{e}=0$ the bound states generally reside at large $\rho / r_{\mathrm{vdW}}$ [4]. The effective range causes an additional repulsive barrier initially leading to less bound energies when $\left|r_{e}\right|$ is small, since the bound state sitting at large $\rho$ will feel the barrier at first. When the effective range increases the attractive region will ultimately lead to an increase in bound state energy as the wave function starts to occupy the pocket at smaller $\rho$.

This is in sharp contrast to the study in Ref. [11] which finds the opposite behavior. As argued above, it is physically reasonable since $E_{T}$ is lower at finite $r_{e}<0$. We notice that our two-channel model has $r_{e}<0$ which is consistent with the usual theory of Feshbach resonances [6]. Ref. [11] appears to accomodate also $r_{e}>0$ and this may resolve the discrepancy.

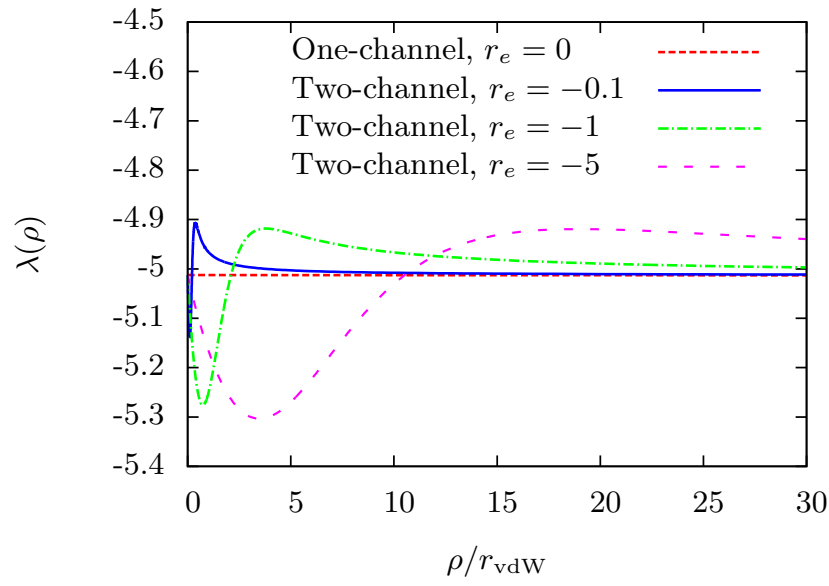

FIG. 3: (Color online) The lowest adiabatic potentials multiplied by $\rho^{2}$ as functions of hyperradius, $\rho$, for the zero range one-channel model and three different effective ranges for the two-channel model.

Connection to two-body potential. The zero-range model studied above does not carry information about the van der Waals length as it stands. However, the three-body parameter or cut-off, $\rho_{c}$, has a physical meaning as it provides a hard-core repulsion in hyperspherical three-body coordinates. To connect the formalism to experimental data, it is therefore necessary to find a relation between the two-body atomic physics and $\rho_{c}$. Below we consider different two-body interaction models and derive analytical formulas for the behavior of $a^{(-)}$as the two-body interaction parameters are varied.

In Fig. 4 we plot Morse, Lennard-Jones, and a van der Waals potential with a hard-core at $r_{c}$. We first focus on the van der Waals plus hard-core model,

$$
V(r)=-\frac{C_{6}}{r^{6}}, \quad r>r_{c}
$$

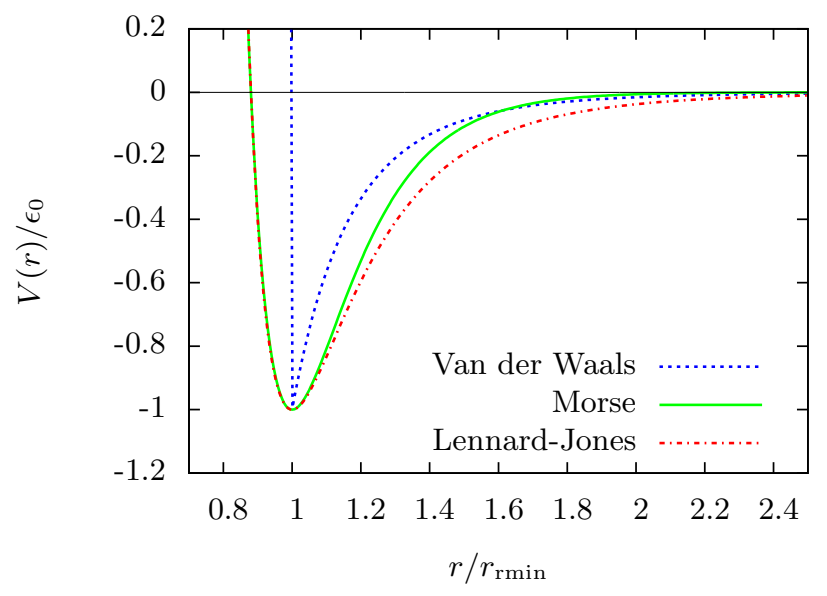

FIG. 4: (Color online) Typical two-body neutral atom-atom potentials, including the hardcore van der Waals potential of Eq. (3), the Lennard-Jones potential, $V_{L J}(r)=\frac{C_{10}}{r^{10}}-\frac{C_{6}}{r^{6}}$, and the Morse potential, $V_{M}(r)=4 \epsilon_{0}\left(e^{-2 \alpha\left(r-r_{0}\right)}-e^{-\alpha\left(r-r_{0}\right)}\right)$. Here $C_{6}, C_{10}$, and $\alpha$ are parameters. They have all been fitted to have the same overall energy, $\epsilon_{0}$, at the radius of the potential minimum, $r_{\min }$.

where $r_{\mathrm{vdW}}=\left(\frac{m C_{6}}{\hbar^{2}}\right)^{1 / 4}$. In order to relate the behavior of this potential to the physics of a Feshbach resonance, we use the formula [16, 18] $a=\bar{a}(1-\tan \Phi)$ with $\Phi=\frac{2 r_{\mathrm{vdW}}^{2}}{r_{c}^{2}}-\frac{3 \pi}{8}$ where $a$ diverges when $\Phi=\left(n+\frac{1}{2}\right) \pi$ for integer $n$, which counts the number of $s$-wave bound states accomodated by the potential. Thus

$$
n=\frac{2}{\pi}\left(\frac{r_{\mathrm{vdW}}}{r_{c}}\right)^{2}-\frac{7}{8}
$$

rounded to highest nearby integer.

In the zero-range single-channel model $\rho_{c}$ and $a^{(-)}$are related, since $\rho_{c}$ is the only length scale available. However, it is a non-trivial matter to determine this relation within the model. Numerically, we find the linear relation $a^{(-)}=-\delta \rho_{c}$, with $\delta=31.756$. To cast this relation into a form that depends only on two-body physics, we observe that the two-body hard-core at $r_{c}$ is also responsible for a three-body hard-core cut-off, more precisely $\rho_{c}=\sqrt{2} r_{c}$. This condition ensures that when each twobody subsystem has radius $r \geq r_{c}$, the third particle will also be outside $r_{c}$ with respect to the others (see Ref. 23] and the supplementary note $\mathrm{A}$ ). We note that this relation is not the same as the $\rho_{c}$ used in Ref. [9]. Using $a^{(-)}=-\delta \rho_{c}$ we obtain

$$
\frac{a^{(-)}}{r_{\mathrm{vdW}}}=-\frac{2 \delta}{\sqrt{\left(n+\frac{7}{8}\right) \pi}} .
$$

This semi-analytical expression for the threshold in terms of the number of bound states is one of our main results.

The relation in Eq. (5) is plotted in Fig. (5) along with the experimental data and the numerical results obtained 


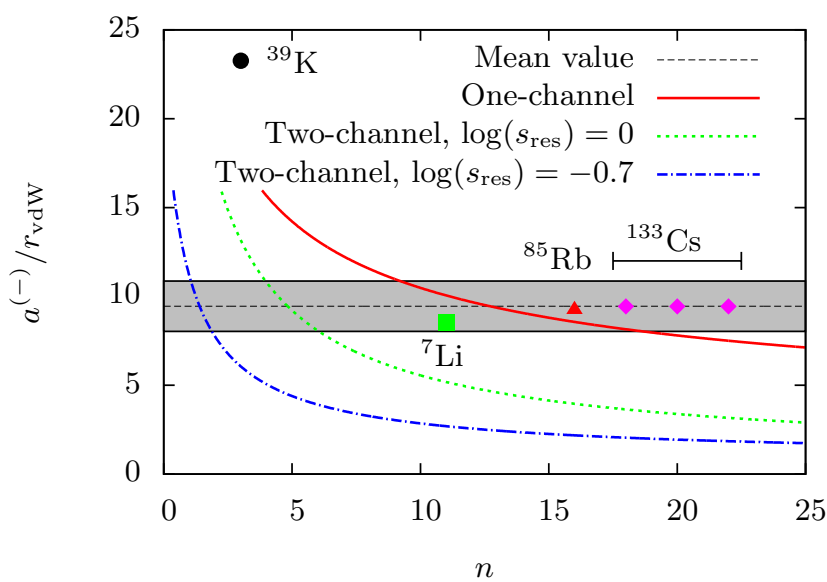

FIG. 5: (Color online) Semianalytic results for $a^{(-)}$plotted against number of bound states in the two-body van der Waals plus hard-core potential. The horizontal position of the experimental data is arbitrary. The grey band indicates the $15 \%$ margin around the mean value of $\sim 9.8$.

from the two-channel model for different value of $s_{\text {res }}$. The one-channel model is consistent with data for $n \sim$ $10-20$ and fits the universal ratio of Ref. 9] for $n=$ 13. This is also consistent with the findings of Ref. 10], although the data only goes to $n=10$. Actually, the $n^{-1 / 2}$ behavior that we find here seems to also appear in Ref. [10], where an extension to higher $n$ could confirm this prediction.

\begin{tabular}{crrrr}
\hline & $\mathrm{Li}$ & $\mathrm{K}$ & $\mathrm{Rb}$ & $\mathrm{Cs}$ \\
\hline$R_{e}[\AA]$ & 2.67 & 3.92 & 4.18 & 4.65 \\
$D_{e}[\mathrm{eV}]$ & 1.06 & 0.52 & 0.49 & 0.45 \\
$n_{L J}$ & 41 & 99 & 152 & 201 \\
$n_{M}$ & 28 & 67 & 103 & 137 \\
\hline
\end{tabular}

TABLE I: Potential parameters, $R_{e}$ (bond length) and $D_{e}$ (dissociation energy) 24], and estimated number of bound states for Lennard-Jones, $n_{L J}$, and Morse, $n_{M}$, potentials.

Even more interestingly, our results for small $s_{\text {res }}$ indicate that $\left|a^{(-)}\right|$drops faster with $n$ than for $s_{\text {res }} \gg 1$. This is seen in the experimental data on ${ }^{7} \mathrm{Li}$ which is slightly below the ${ }^{85} \mathrm{Rb}$ and ${ }^{133} \mathrm{Cs}$ points, but our model seems to overestimate this trend. Clearly, more results on narrow resonance systems are required to address the question of effective range corrections. We expect a lower $\left|a^{(-)}\right|$value than for broad resonances.

Two-body potential models Above we employed a van der Waals plus hard-core potential. However, as seen in Fig. 4. more realistic Lennard-Jones or Morse potentials have a smooth behavior of the inner barrier. This implies only minor quantitative corrections that nevertheless deserve to be addressed along with the number of bound states expected in a real alkali dimer.
The number of $s$-wave bound states in the LennardJones (LJ) and Morse (M) potentials can be estimated analytically and yields [25] $n_{L J}=0.361 \sqrt{\beta}-\frac{5}{8}$ and $n_{M}=$ $0.245 \sqrt{\beta}-\frac{1}{2}$, where $\beta=\frac{m r_{\min }^{2} \epsilon_{0}}{2 \hbar^{2}}$ with $r_{\min }$ the radius at which the potential takes its minimal value, $\epsilon_{0}$. For comparison, the expression in Eq. (4) can be written $n=$ $0.225 \sqrt{\beta}-\frac{7}{8}$, with $r_{\min } \leftrightarrow r_{c}$ in $\beta$. The similarity of these expressions makes it clear that the behavior seen in Fig. [5] is generic and does not depend on our choice of two-body potential. The difference in constant provides only a minor quantitative change in the numbers.

An important question, however, remains about the number of bound states in a real alkali dimer system. Using the molecular dissociation energy, $\epsilon_{0}=D_{e}$, and the bond length, $r_{\min }=R_{e}$, of Ref. [24], we list the numbers for $\mathrm{Li}, \mathrm{K}, \mathrm{Rb}$, and $\mathrm{Cs}$ dimers in Tab. II. The estimated number of bound states is outside the axis in Fig. 5 and also much beyond the results shown in Ref. [10]. The agreement with theory at a rather limited number of bound states $(n \sim 10-20)$ is then quite surprising.

A number of important observations can be made. First, the decrease of $\left|a^{(-)}\right|$with $n$ is weak, and a shift of the length scale in Fig. [5 would therefore place the onechannel model within the experimental range for larger $n$ and it would stay within the $15 \%$ deviation from the mean for a larger interval (since the slope at larger $n$ decreases even faster). Second, the experimental data might indicate that only a certain number of bound states play an active role. Equivalently, even if the two-body potential is very deep, only the upper part of the twobody potential and the bound states closest to threshold set the scale of the three-body problem. This appears to be very reasonable since we are considering universal Efimov trimers here and not strongly bound three-body states. Third, the case of small $s_{\text {res }}$ has $\left|a^{(-)}\right| \propto n^{-r}$ with $r>1 / 2$ as seen in Fig. 5. This implies that narrow resonance systems should be even more insensitive to $n$ beyond a certain lower limit.

We can give a quantitative argument for the lack of sensitivity to the many deeper bound states in a van der Waals potential. The number of bound states, $n(E)$ as a function of energy, $E$, counted from the $E=0$ threshold and down within the WKB approximation is $n(E)=n_{0}\left(|E| / E_{\mathrm{vdW}}\right)^{1 / 3}$, where $n_{0}$ is the total number (given in Tab. I for different potentials) and $E_{\mathrm{vdW}}=$ $\hbar^{2} / m r_{\mathrm{vdW}}^{2}>0$ is the depth of the potential [26]. For the $s_{\text {res }} \gg 1$ cases $\left({ }^{85} \mathrm{Rb}\right.$ and $\left.{ }^{133} \mathrm{Cs}\right), n(E) / n_{0} \sim 0.10-0.20$ which implies $|E| / E_{\mathrm{vdW}} \sim 0.001-0.01$. Numerically we find a three-body energy on resonance $E_{3}=0.006 E_{\mathrm{vdW}}$ (using $\rho_{c}=0.58 r_{\mathrm{vdW}}$ ). However, universality relates $E_{3}=\hbar^{2} \kappa^{2} / m$ and $a^{(-)} \kappa \sim-1.51[1,5]$. The energy scale at the continuum threshold is given by $a^{(-)}$through $|E| \sim \hbar^{2} / m\left(a^{(-)}\right)^{2}=0.003 E_{\mathrm{vdW}}$, in agreement with the interval above. In the case of ${ }^{7} \mathrm{Li}, E_{3}$ is similar but this is compensated by a smaller $n_{0}$ so this case can also be explained. For heavier alkali atoms with narrow resonance, our two-channel results predict a smaller $a^{(-)} / r_{\mathrm{vdW}}$ than 9.8 , which is a good experimental test of our theory. 
a)

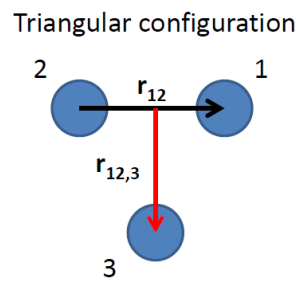

b)

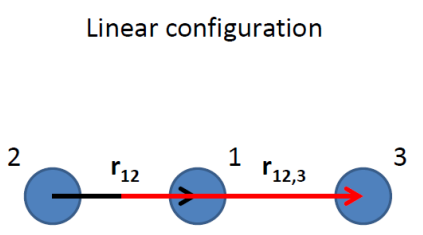

FIG. 6: (Color online) Schematic of the a) triangular and b) linear configurations for an equal mass three-body system. $\boldsymbol{r}_{12}$ and $\boldsymbol{r}_{12,3}$ indicate the two Jacobi relative vectors.

Conclusions. We derive an analytical formula that connects $a^{(-)}$and the number of $s$-wave bound states, $n$, in the two-body potential which agrees with recent experiments $n \sim 10-20$. While alkali atoms typically have larger $n$, we argue quantitatively that only a subset determine the properties of Efimov three-body states. We find non-monotonic behavior of $a^{(-)}$with increasing effective range which demonstrates that systems with narrow Feshbach resonances will provide both qualitative and quantitative understanding of universality and the origin of the three-body parameter. This means that different atomic species which typically have narrow resonances and a denser spectrum would be very helpful.

Acknowledgments We thank Chris Greene, Georg Bruun, and Frank Jensen for useful discussions. This work is supported by the Danish Council for Independent Research - Natural Sciences (DFF-FNU).

\section{Appendix A: Determination of the hyperradial boundary condition}

The atom-atom two-body potential has a steep repulsive inner core which we will assume for the moment can be representated by a hard wall, i.e. an infinite potential for radii $r<r_{c}$. In this case the boundary condition is simply that the two-body wave function must be zero at $r_{c}$. This must then be translated in the three-body problem where it implies that the total wave function must be zero whenever any of the relative distances between two out of the three atoms is less than or equal to $r_{c}$. Any penetration of the wave function into the wall would cost an infinite amount of energy and is thus forbidden.

A neat and elegant way to obtain a condition on $\rho$ is the following. As is easily shown the hyperradius fulfills

$$
\rho^{2}=\frac{1}{3} \sum_{i<k}\left(\boldsymbol{r}_{i}-\boldsymbol{r}_{k}\right)^{2}=\frac{1}{2} \boldsymbol{r}_{12}^{2}+\frac{1}{3} \boldsymbol{r}_{12,3}^{2},
$$

where $\boldsymbol{r}_{12}=\boldsymbol{r}_{1}-\boldsymbol{r}_{2}$ and $\boldsymbol{r}_{12,3}=\boldsymbol{r}_{3}-\left(\boldsymbol{r}_{1}+\boldsymbol{r}_{2}\right) / 2$ are respectively the relative vector from particles 2 to 1 and the relative vector from the center of mass of 1 and 2 to particle 3 (see Fig [6).

Now consider the triangular configuration shown in a) of Fig. 6. From the formula in Eq. (A1), it is clear that when $r_{12}=r_{c}$ this setup also has $\rho=r_{c}$. However, the linear configuration of b) in Fig. 6 with $\rho=r_{c}$ will then have two atoms that are within the hard-core radius and yield an infinite contribution to the potential energy (this happens f.x. when $|\boldsymbol{x}|=r_{c}$ and $\boldsymbol{y}=0$ putting atom 3 midway between 1 and 2).

Consider instead the linear configuration with $r_{12}=r_{c}$ and impose the requirement $r_{23} \geq r_{c}$, where $\boldsymbol{r}_{23}=\boldsymbol{r}_{2}-$ $\boldsymbol{r}_{3}$. Since we have $\boldsymbol{r}_{12,3}=\boldsymbol{r}_{23}+\boldsymbol{r}_{12} / 2$ we get

$$
\rho^{2} \geq r_{c}^{2}\left(\frac{1}{2}+\frac{2}{3}\left[1+\frac{1}{2}\right]^{2}\right)=2 r_{c}^{2}
$$

We thus see that the condition $\rho>\sqrt{2} r_{c}$ ensures that both triangular and linear configurations are outside the hard-core regions. Since these configurations are extremal, the condition implies that no regions with infinite potential are sampled by the hyperradial three-body wave function.

The rigourous formal argument for the validity of the relation $\rho_{c}=\sqrt{2} r_{c}$ using the hyperspherical approach can be found in Ref. 23], where the relation is derived using a square well potential. The asymptotic region is precisely $\rho>\sqrt{2} r_{c}$ as found above. Here we assumed a hard-core potential for simplicity which gives the factor of $\sqrt{2}$. For a real atom-atom potential, the hard-core is slightly softer (typically of the $1 / r^{12}$ ) which may lead to a minor change in the factor.

The hyperspherical approach and the use of the lowest hyperradial potential to describe Efimov states is a well-known and accurate procedure. Additionally, as we have shown above, a hard-core repulsion in the two-body potential naturally generates a hyperspherical cut-off radius, $\rho_{c}=\sqrt{2} r_{c}$. This constitutes a clear and simple explanation of the repulsive core found in the numerical calculations in Ref. [10], and our results demonstrate that a simple model can accurately describe those numerically involved findings. We thus see how the value $\sqrt{2} r_{c}$ for the three-body cut-off parameter naturally derives from the hard-core behavior of the corresponding two-body potential.
[1] V. Efimov, Yad. Fiz 12, 1080 (1970); Sov. J. Nucl. Phys. 12, 589 (1971).
[2] A. S. Jensen, K. Riisager, D. V. Fedorov, and E. Garrido, Rev. Mod. Phys. 76, 215 (2004). 
[3] F. Ferlaino and R. Grimm, Physics 3, 9 (2010).

[4] E. Nielsen, D. V. Fedorov, A. S. Jensen, and E. Garrido, Phys. Rep. 347, 373 (2001).

[5] E. Braaten and H. W. Hammer, Phys. Rep. 428, 259 (2006).

[6] C. Chin, R. Grimm, P. S. Julienne, and E. Tiesinga, Rev. Mod. Phys. 82, 1225 (2010).

[7] M. Berninger et al., Phys. Rev. Lett. 107, 120401 (2011).

[8] P. Naidon, E. Hiyama, and M. Ueda, Phys. Rev. A 86, 012502 (2012).

[9] C. Chin, arXiv:1111.1484v2.

[10] J. Wang, J. P. D'Incao, B. D. Esry, and C. H. Greene, Phys. Rev. Lett. 108, 263001 (2012).

[11] R. Schmidt, S. P. Nath, and W. Zwerger, arXiv:1201.4310v2

[12] T. Kraemer et al., Nature 440, 315 (2006).

[13] S. E. Pollack, D. Dries, and R. G. Hulet, Science 326, 1683 (2009).

[14] M. Zaccanti et al., Nature Phys. 5, 586 (2009).

[15] R. J. Wild, P. Makotyn, J. M. Pino, E. A. Cornell, and D. S. Jin, Phys. Rev. Lett. 108, 145305 (2012).

[16] G. F. Gribakin and V. V. Flambaum, Phys. Rev. A 48, 546 (1993)

[17] B. Gao, Phys. Rev A 58, 4222 (1998).
[18] V. V. Flambaum, G. F. Gribakin, and C. Harabati, Phys. Rev. A 59, 1998 (1999)

[19] Y. Suzuki and K. Varga, Stochastic Variational Approach to Quantum-Mechanical Few-Body Problems, (SpringerVerlag, Berlin Heidelberg, 1998).

[20] P. K. Sørensen, D. V. Fedorov, and A. S Jensen, FewBody Systems, 0177-7963 (2012)

[21] N. Gross, Z. Shotan, S. Kokkelmans, and L. Khaykovich, Phys. Rev. Lett. 103, 163202 (2009).

[22] N. Gross, Z. Shotan, S. Kokkelmans, and L. Khaykovich, Phys. Rev. Lett. 105, 103203 (2010).

[23] A. S. Jensen, E. Garrido, and D. V. Fedorov, Few-Body Syst. 22, 193 (1997).

[24] Igel-Mann et al, J. Chem. Phys., 849 (1986)

[25] G. D. Mahan, M. Lapp, Phys. Rev., 1791 (1969)

[26] The number of bound states, $n(E)$, can be found within the WKB formalism by considering the integral $\int_{r_{i}}^{r_{o}} \sqrt{E-V(r)} d r=\pi \hbar(n(E)+1 / 4)$ which is a measure of the number of nodes in the wave function with energy $E<0$. Using $E=0$ one obtains the total number, $n_{0}$. The van der Waals potential does not yield analytical results, but we numerically find that $n(E) \propto|E|^{1 / 3}$, which when normalized yields the relation in the text. 